\title{
Anopheles aquasalis transcriptome reveals autophagic responses to Plasmodium vivax midgut invasion
}

Rosa Amélia Gonçalves Santana ${ }^{1}$, Maurício Costa Oliveira ${ }^{1}$, Iria Cabral' ${ }^{1}$ Rubens Celso Andrade Silva Junior ${ }^{1}$, Débora Raysa Teixeira de Sousa', Lucas Ferreira', Marcus Vinícius Guimarães Lacerda,2,

Wuelton Marcelo Monteiro' ${ }^{1}$ Patrícia Abrantes ${ }^{3}$, Maria das Graças Vale Barbosa Guerra ${ }^{1}$ and Henrique Silveira ${ }^{1,3^{*}}$

\begin{abstract}
Background: Elimination of malaria depends on mastering transmission and understanding the biological basis of Plasmodium infection in the vector. The first mosquito organ to interact with the parasite is the midgut and its transcriptomic characterization during infection can reveal effective antiplasmodial responses able to limit the survival of the parasite. The vector response to Plasmodium vivax is not fully characterized, and its specificities when compared with other malaria parasites can be of fundamental interest for specific control measures.

Methods: Experimental infections were performed using a membrane-feeding device. Three groups were used: $P$. vivax-blood-fed, blood-fed on inactivated gametocytes, and unfed mosquitoes. Twenty-four hours after feeding, the mosquitoes were dissected and the midgut collected for transcriptomic analysis using RNAseq. Nine cDNA libraries were generated and sequenced on an Illumina HiSeq2500. Readings were checked for quality control and analysed using the Trinity platform for de novo transcriptome assembly. Transcript quantification was performed and the transcriptome was functionally annotated. Differential expression gene analysis was carried out. The role of the identified mechanisms was further explored using functional approaches.
\end{abstract}

Results: Forty-nine genes were identified as being differentially expressed with P. vivax infection: 34 were upregulated and 15 were downregulated. Half of the P. vivax-related differentially expressed genes could be related to autophagy; therefore, the effect of the known inhibitor (wortmannin) and activator (spermidine) was tested on the infection outcome. Autophagic activation significantly reduced the intensity and prevalence of infection. This was associated with transcription alterations of the autophagy regulating genes Beclin, DRAM and Apg8.

Conclusions: Our data indicate that P. vivax invasion of An. aquasalis midgut epithelium triggers an autophagic response and its activation reduces infection. This suggests a novel mechanism that mosquitoes can use to fight Plasmodium infection.

Keywords: Malaria transmission, Anopheles mosquitoes, Host parasite interactions, Plasmodium vivax, Malaria control, Autophagy

\footnotetext{
*Correspondence: hsilveira@ihmt.unl.pt

${ }^{3}$ Instituto de Higiene e Medicina Tropical, Global Health and Tropical

Medicine, Universidade Nova de Lisboa, Lisboa, Portugal

Full list of author information is available at the end of the article
} 


\section{Background}

Malaria is still an important public health problem in several tropical countries. In 2016, 216 million cases of malaria were diagnosed; Brazil had $18 \%$ of all malaria cases that were confirmed by the World Health Organization (WHO) for the region of the Americas [1], and 99.5\% of the Brazilian cases were in the Legal Amazon [2] where Plasmodium vivax is the predominant species accounting for $85 \%$ of reported cases [3].

Anopheles aquasalis is an important vector in coastal areas of South America [4,5]. Since its colonization it has been used for Plasmodium experimental infections for research, revealing a robust model to study the interaction of American vectors with Plasmodium species [6].

Inside the mosquito midgut the Plasmodium gametocyte-to-ookinete-to-oocyst transition is completed within the first 24 hours. The ingested parasite populations suffer substantial losses during this process which corresponds to the most critical population bottleneck of the entire parasite life-cycle; more often than not, transmission is terminated at this stage [7-9]. Invasion of the malaria vector Anopheles gambiae midgut by Plasmodium parasites triggers transcriptional changes of genes that mediate the antiparasitic defence [10] and, thus, the ability of these mosquitoes to transmit malaria [11]. Moreover, several mechanisms are triggered by the mosquito in order to combat the intracellular pathogen. Apoptosis is one possibility that has been described during ookinete invasion of the midgut [12]. Another related mechanism is autophagy, an important and well-studied cytosolic response. During macroautophagy, a double membrane vesicle called autophagosome forms around cytosolic components, which subsequently fuse with lysosomes and degrade the vessel's content [13]. Under certain conditions in Drosophila, midgut and salivary gland tissues show both high caspase activity and the formation of autophagosomes [14, 15], suggesting that apoptosis and autophagy may be highly integrated in arthropods.

The upregulation of autophagy can enhance resistance to pathogens, a phenomenon that has also been associated with resistance of the fruit fly Drosophila melanogaster to bacterial challenge [16] and of mammalian cells to bacteria, viruses and parasites such as Toxoplasma gondii [17-21]. Intriguingly, a $P$. falciparum infection in Anopheles stephensi induced a translation of autophagy (ATG) protein mRNAs, including those for key regulators ATG6 and ATG8, in the midgut epithelium at 24 hours after infection [22], which suggests that autophagy is induced early during sporogonic development in the mosquito host. While autophagy induction can control resistance, perhaps contributing to the large-scale death of parasites in the midgut, it is possible that highly conserved regulation of stem cell renewal and differentiation by autophagy could also impact the midgut's response to parasite infection [23].

Transcriptomic analyses of African and Asian mosquitoes in response to pathogens have generated a wealth of data that can facilitate the development of new anti-malaria tools [24, 25]. More recently, $A n$. aquasalis specimens have been analysed for functional annotation creating opportunities for further molecular characterization of genes. The An. aquasalis transcriptomes of larvae and adults fed on sugar and on blood revealed valuable information about protein-coding transcripts involved in biological processes relevant to mosquito physiology and development of this new world model [26].

Nevertheless, a deeper understanding of the processes taking part on this critical phase of malaria transmission remains unexplored. Here, we report the transcriptional profile of $A n$. aquasalis midgut, in the early stage of $P$. vivax development and invasion of the midgut epithelium. Via this profile, we were able to gain insights on a molecular level of how to functionally characterize this critical phase of malaria transmission. Our results revealed the importance of alternative mechanisms, such as autophagy, for the control of Plasmodium infection in the mosquito.

\section{Methods}

\section{Mosquito collections and rearing}

An. aquasalis were obtained from a well-established colony at the Entomology Department's insectary at the Fundação de Medicina Tropical Dr Heitor Vieira Dourado, Manaus, Amazonas, Brazil (FMT-HVD). All mosquitoes were reared at $26{ }^{\circ} \mathrm{C}, 70-80 \%$ relative humidity under a 12/12 light/dark photoperiod. Larvae were fed with commercial fish food (Tetramin Gold ${ }^{\circledR}$; Tetra GmbH, Melle, Germany) and adults were fed $a d$ libitum on $10 \%$ sugar solution. Three- to five-day-old adult females were used in all experiments.

\section{Blood collection}

Adult volunteers (ages $\geq 18$ years), residents from the region of Manaus (State of Amazonas, Brazil) with $P$. vivax malaria infection diagnosed by blood smears, were recruited at the Fundação de Medicina Tropical Dr Heitor Vieira Dourado (FMT HVD). All volunteers were instructed on the study objectives. A sample of about $10 \mathrm{ml}$ of venous blood was drawn from each patient and placed in heparinized tubes. After blood collection all patients were treated according to Brazilian Health Ministry guidelines [27]. 


\section{Plasmodium vivax infection of mosquitoes via membrane feeding assay}

Adult mosquitoes were sugar starved overnight prior to infection and separated into two experimental groups. One group was offered whole blood from $P$. vivax patients for a period of 45-90 min via membrane feeding assay (MFA). Blood was held at $37^{\circ} \mathrm{C}$ through a hose system connected to a thermal bath [6]. The second group was treated in similar way, but with inactivated gametocytes as described by Mendes et al. [28]: P. vivax infective blood was briefly heated for $15 \mathrm{~min}$ at $43{ }^{\circ} \mathrm{C}$, chilled to $37{ }^{\circ} \mathrm{C}$ and then offered to the mosquitoes. Only fully engorged mosquitoes were transferred to rearing containers and maintained in the insectary at $26{ }^{\circ} \mathrm{C}$ with $70-80 \%$ relative humidity and fed ad libitum on $10 \%$ sugar solution.

\section{Tissue collection and RNA isolation}

Mosquito midguts were collected from pools of 30 mosquitoes, 18-24 h after the blood meal, from each of three groups: (i) P. vivax-blood-fed group $(P v)$; (ii) non-infective group ( $B l$; blood-fed using $P$. vivax-blood in which the gametocytes were inactivated); and (iii) unfed group (Unf; unfed mosquitoes). Tissues were dissected from mosquitoes submerged in ice-cold phosphate buffered saline (PBS) and transferred to RNAlater (Thermo Fisher Scientific, Massachusetts, USA). Samples were stored at $-20{ }^{\circ} \mathrm{C}$ until RNA extraction. Total RNA was extracted using TRIzol Reagent (Thermo Fisher Scientific, Massachusetts, USA) following the manufacturer's protocol. To eliminate possible contaminant genomic DNA, the RNA samples were treated with RNase-Free DNase I according to manufacturer's protocol (Qiagen, Hilden, Germany). At 8-9 days post-infection, mosquito midguts were collected to determine the infection rate (number of infected mosquitoes over total number of mosquitoes observed) and infection intensity (mean number of oocysts per infected mosquito). Three independent biological replicates of each experiment were performed.

\section{RNA-seq library preparation and sequencing}

The RNA integrity was confirmed using a 2100 Bioanalyzer (Agilent, California, USA). The RNA-seq library preparation and sequencing were performed using total RNA and an Illumina $\mathrm{HiSeq}^{\circledR} 2500$ (Illumina, California, USA) at LaCTAD [Life Sciences Core Facility from State University of Campinas (UNICAMP); https://www. lactad.unicamp.br]. Illumina reads from the $A n$. aquasalis mosquitoes were checked for quality control using FastQC v.0.11.5 (https://www.bioinformatics.babraham. ac.uk) and analysed using the Trinity platform for de novo transcriptome assembly v.2.4.0 [29]. Trimmomatic was used to trim low-quality reads and high quality pairedend reads were assembled using Trinity Assembler v2.4.0 and aligned using Bowtie2 v.2.3.2 [30]. Transcript quantification was performed using RSEM module v.1.2.25 [31]. Transdecoder v.3.0, Trinotate v.3.0.2, BLAST + (accessed on 2017/04/26) and HMMER v.3.0 searches were used for functional annotation of the transcriptome produced and to populate a Sqlite database.

Differential expression (DE) analysis was performed using GLM test in the edgeR v.3.16.5 package [32] in R. Pairwise comparisons were made between the different group samples. In any given group, a transcript was considered differentially expressed if its adjusted $P$-value to control the false discovery rate (Benjamini-Hochberg adjustment) was less than 0.05 and if log fold change was higher than 1. Differentially expressed genes were further analysed for functional classification using gene ontology analysis on PANTHER (http://www.pantherdb.org) [33]. The dataset has been deposited at the Gene Expression Omnibus under the accession number GSE124997.

\section{Validation of differentially expressed genes}

In order to validate transcriptome analysis, a total of 8 differentially expressed genes between mosquitoes fed on blood with infective $P$. vivax $(P v)$ and fed on blood in which gametocytes were inactivated $(B l)$ were chosen for real-time quantitative PCR analysis which was performed as described in [34]. For this, total RNA was used, and first strand cDNA was synthesized using oligo $\mathrm{dT}$ and MMLV Reverse Transcriptase (Promega, Wisconsin, EUA) as described in [35]. The cDNA was used as a template for RT-qPCRs using the primer pairs reported in Additional file 1: Table S1. The primers were derived from the sequences identified in the transcriptome. For all groups, mosquito midguts were collected $18-24 \mathrm{~h}$ post-infection in order to determine the levels of expression of the genes in the midgut. Experiments were conducted with three biological replicates, each in triplicate.

Reverse transcription quantitative real-time PCR (RT-qPCR) Real-time quantitative PCR was performed on a Fast 7500 instrument (Applied Biosystems, California, USA) with SYBR Green Power Master Mix (Applied Biosystems) using $2 \mu \mathrm{l}$ of cDNA template in a final volume of $20 \mu \mathrm{l}$ reaction mixture. Fold-changes of gene expression were analysed using the $2^{-\Delta \Delta C T}$ method. The ribosomal protein S7 was used as the endogenous control.

\section{Spermidine and wortmannin treatments}

The transcriptome associated to $P$. vivax infection revealed a variety of transcripts that play a key role in autophagy. In order to evaluate the effect of the autophagy process in the outcome of infection, we 
inoculated mosquitoes with wortmannin (an inhibitor of phosphatidylinositol 3-kinase DPI3K) and spermidine (an autophagy activator) [36, 37]. Three- to four-day-old female mosquitoes were cold-anesthetized and inoculated intrathoraxically with $69 \mathrm{nl}$ of a $5 \mu \mathrm{M}$ and $0.05 \mu \mathrm{M}$ solution of wortmannin (Merck, Darmstadt, Germany) or with the same volume of $\mathrm{H}_{2} \mathrm{O}$ Ultra Pure and with 69 $\mathrm{nl}$ of a $100 \mu \mathrm{M}$ solution of spermidine (Sigma) or DMSO $(0.05 \%)$ using a Nanoject micro-injector (Drummond Scientific, Pennsylvania, USA). Twenty-four hours after injection with the solutions, the mosquitoes were fed with a $P$. vivax-infected blood meal as described above. Three independent biological replicates were performed for each experiment. Mosquitoes were dissected 18-24 $\mathrm{h}$ after feeding; batches of 20-30 midguts were dissected in cold DEPC-treated phosphate-buffered saline (PBS) and processed for RNA preparation and cDNA synthesis using the same protocols mentioned above. Mosquito midguts were also collected on the 8th day post-infection to determine the prevalence and intensity of infection.

\section{Gene expression of autophagy related genes}

The expression of genes that regulate autophagy (Beclin, DRAM and Apg8) was investigated 18-24 h after $P$. vivax infection and $24 \mathrm{~h}$ after inhibition and activation of autophagy (treatment with wortmannin or spermidine) as described above.

Anopheles aquasalis mosquitoes were dissected 18-24 $\mathrm{h}$ after infection; 20-30 midguts were collected, and RNA and cDNA were prepared as described above. Three independent experiments were performed. Gene expression analysis was carried out by quantitative real-time PCR following the same conditions described above.

\section{Statistical analysis}

For data not normally distributed (oocyst density), twosample comparisons were done using the non-parametric Mann-Whitney test. The differences in the infection rate between the control group and the tested groups were compared using Fisher's exact one-tailed test (F). Comparisons of mRNA expression levels obtained by RTqPCR between the control and the tested groups were done using the Mann-Whitney one-tailed test. Statistical analyses were performed using the software GraphPad Prism v.6.00.

\section{Results and discussion}

\section{General characterization of midgut transcriptome}

A total of $9 \mathrm{cDNA}$ libraries from An. aquasalis midguts were constructed and sequenced, namely three libraries for each of the following groups: (i) $P$. vivax-blood-fed mosquitoes ( $P v$ : groups $P v 1$ to $P v 3$ ); (ii) mosquitoes fed on $P$. vivax-blood from which gametocytes were inactivated (non-infected: groups $B l 1$ to $B l 3$ ); and (iii) unfed mosquitoes (unfed groups: Unf1 to Unf3). The obtained mean number of high quality paired-end short reads were: 56,217,833 (16,351,414-109,481,490), 45,546,489 $(44,961,578-46,664,218)$ and 46,523,955 (43,800,760$50,915,130)$ for each group, respectively (Table 1 ).

To examine differential expression between mosquitoes fed on $P$. vivax infected-blood $(P v)$ and mosquitoes fed on non-infective blood $(\mathrm{Bl})$ or unfed mosquitoes, FDR $<0.05$ and $\operatorname{logFC}>1$ (fold change) were used as the threshold to classify differentially expressed genes. The analyses showed a total of 12,942 expressed genes. Of these, 49 genes were identified as differentially expressed genes in the $P$. vivax infected-blood-fed group $(P v)$ in relation to non-infected-blood-fed group $(B l)$; 34 were upregulated and 15 were downregulated, which represents differential expression associated to $P$. vivax infection (Fig. 1). A total of 111 genes were differentially expressed in infectedblood-fed mosquitoes $(P v)$ when compared to the unfed group (Unf); of these, 65 were upregulated and 46 were downregulated (Fig. 2). The detailed gene lists are shown in Additional file 2: Tables S2-S5.

About $49 \%$ (24 out of 49) of the differentially expressed genes in the $P v \times B l$ group were exclusive to this comparison (Fig. 3) and involved a large gene set related to autophagy. On the other hand, the $P v \times U n f$ comparison presented 86 out of 111 (78\%) of the differentially expressed genes exclusively in this group (Fig. 3).

To validate the robustness of RNAseq results, we analysed eight genes by real-time qRT-PCR and compared the expression of these genes in $\mathrm{Pv} \times \mathrm{Bl}$ (Additional file 3: Figure S1). These analyses revealed a significant correlation (Pearson's correlation coefficient $=0.874$, $R^{2}=0.7663$, slope $\left.=0.04539\right)$ between the qRT-PCR and the RNAseq data.

Table 1 Overview of Anopheles aquasalis sequencing results

\begin{tabular}{lll}
\hline & No. of reads & $\%$ bases $\geq$ Q30 \\
\hline P. vivax blood meal $(P v)$ & & \\
Pv1 & $44,961,578$ & 93.16 \\
Pv2 & $45,013,670$ & 90.99 \\
Pv3 & $46,664,218$ & 93.28 \\
Non-infective blood meal (Bl) & & \\
Bl1 & $42,820,594$ & 92.15 \\
Bl2 & $16,351,414$ & 93.02 \\
Bl3 & $109,481,490$ & 93.37 \\
Unfed (Unf) & & \\
Unf1 & $43,800,760$ & 91.70 \\
Unf2 & $44,855,976$ & 93.36 \\
Unf3 & $50,915,130$ & 90.90 \\
\hline
\end{tabular}




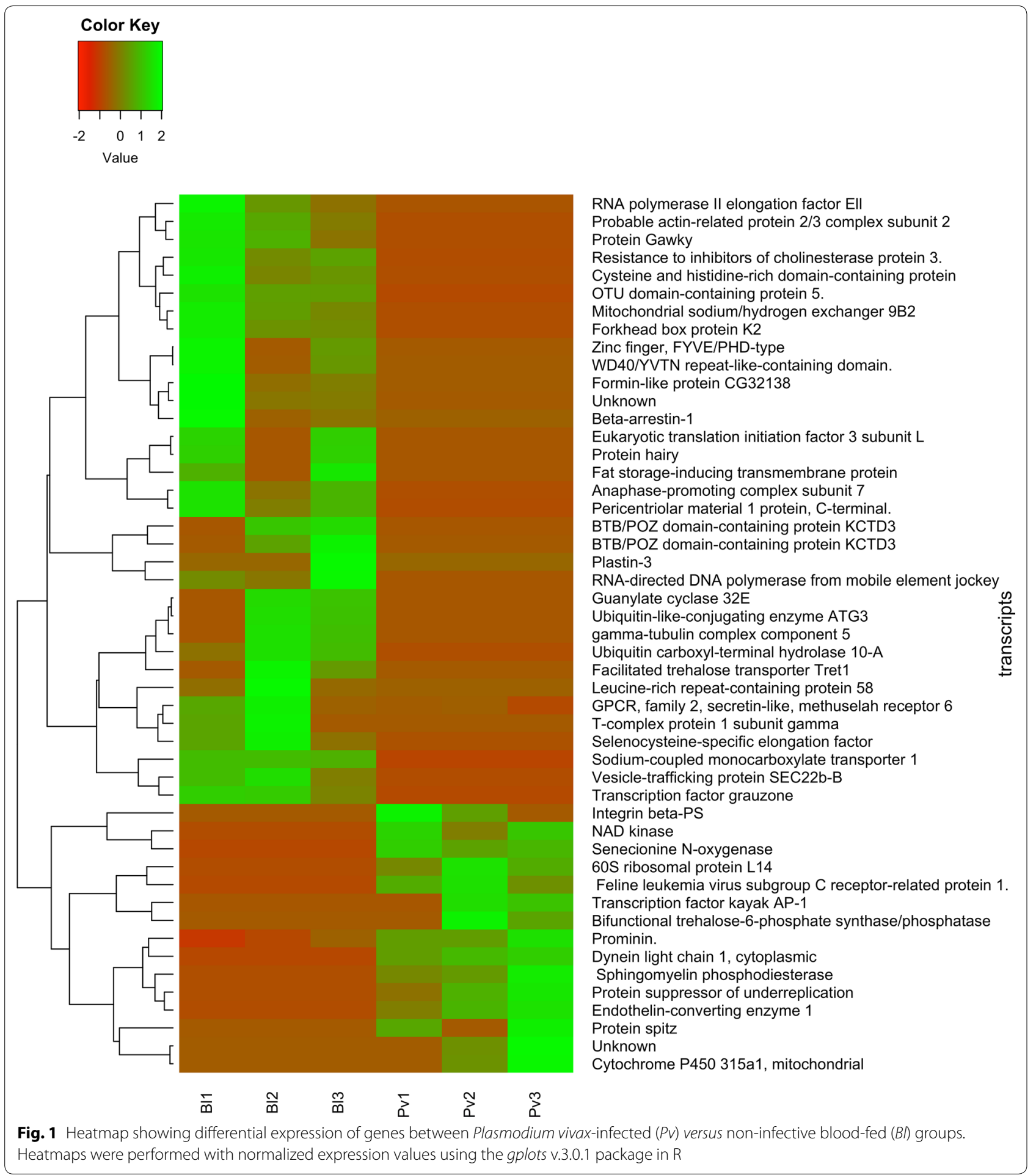




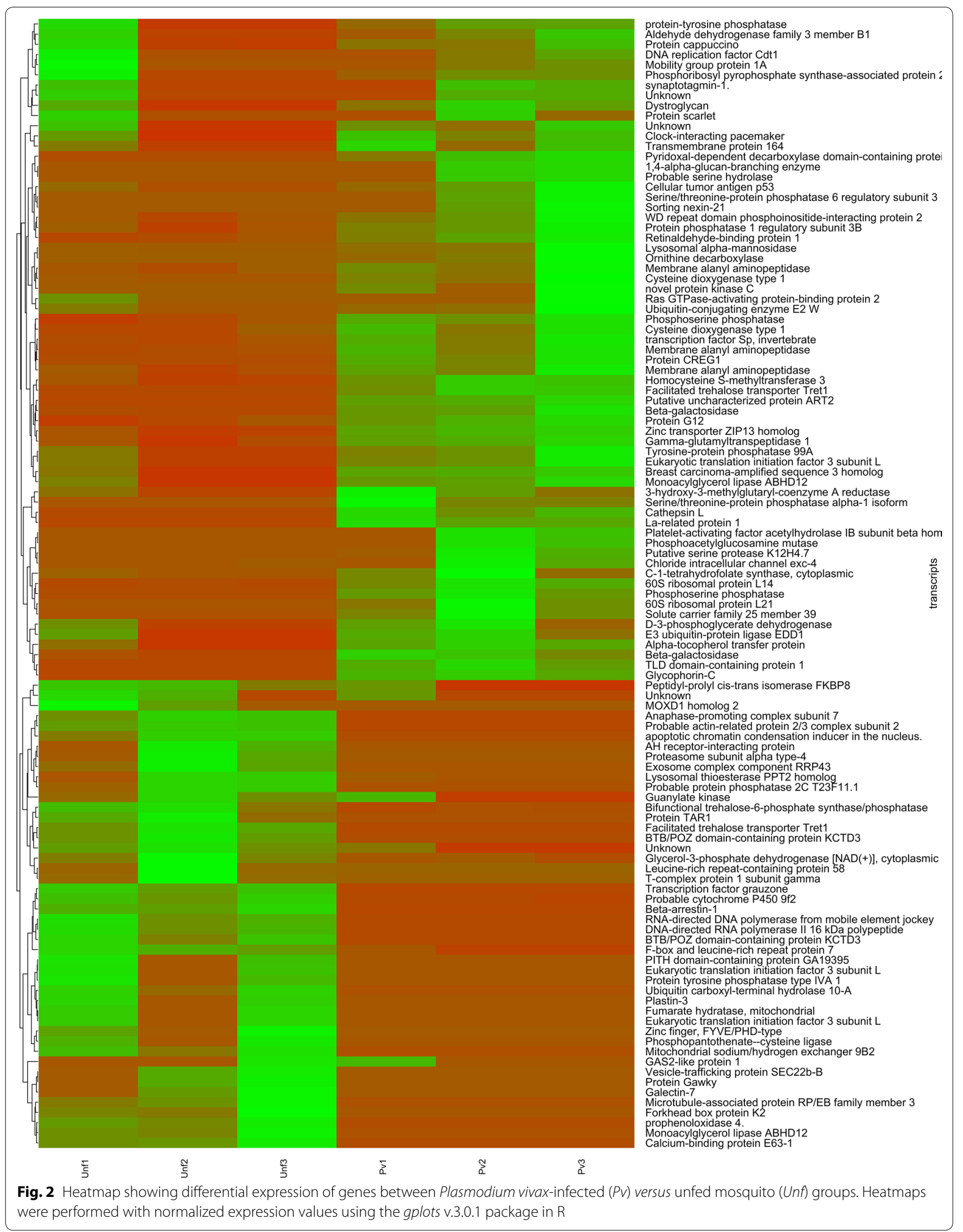



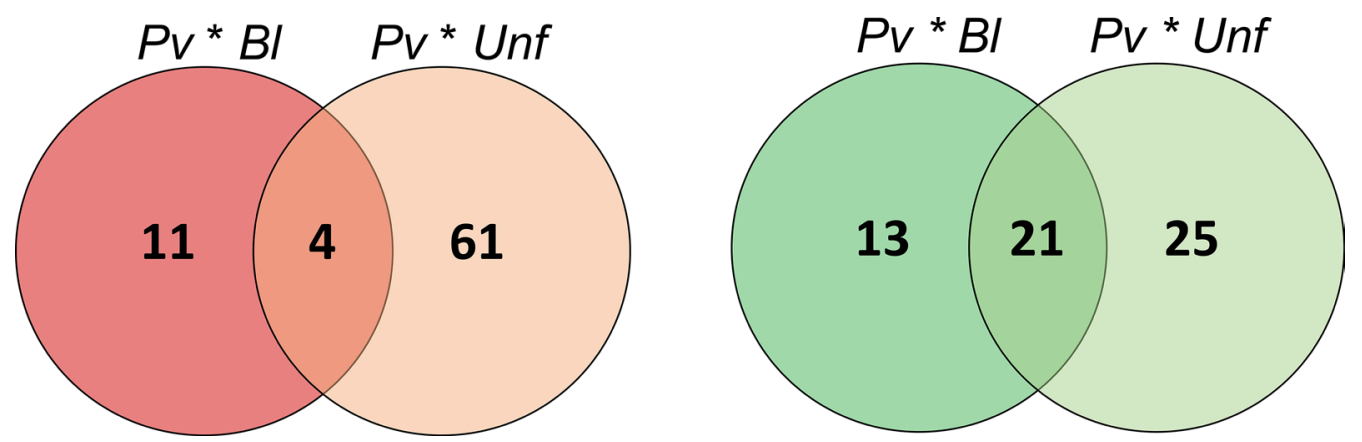

Fig. 3 Proportions of Anopheles aquasalis midgut differentially expressed genes within different groups comparison. a Upregulated genes. $\mathbf{b}$ Downregulated genes. Abbreviations: Pv, P. vivax blood-meal group; Bl, non-infective blood-meal group; Unf, unfed group

\section{Anopheles aquasalis midgut differential gene expression associated with Plasmodium vivax infection}

Transcriptomic analysis of midgut infected mosquitoes revealed 49 differentially expressed genes in the $P$. vivax infected-blood-fed group $(P v)$ in relation to noninfected-blood-fed group $(B l)$; of these, 34 were upregulated and 15 were downregulated. From these, genes involved in cellular process, metabolic process (GO: 0008152), cellular component organization or biogenesis process (GO: 0050896) and biological regulation process (GO: 0065007) were predominant (Fig. 4). The results suggest that many of the upregulated genes are involved in several metabolic processes and molecular functions, among them, catalytic activity (GO: 0003824) and cofactor binding (GO: 0005488) were enriched with hits of 6 and 4 genes, respectively, followed by transporter and structural molecule activity.

\section{Immunity}

Activation of mosquito immunity genes has been traditionally associated with midgut-infected mosquitoes. In the present study, a transcript coding for a leucinerich repeat protein, orthologue of LRR-containing protein 58 (TRINITY_DN6165_c5_g1_i4), was found upregulated in mosquitoes infected with $P$. vivax $(P v)$ when compared to the mosquitoes fed on inactivated gametocytes $(\mathrm{Bl})$. LRR-containing protein 58 has been previously associated with the An. gambiae response to Plasmodium berghei infection [38, 39]. LRR containing proteins are related to the control of the complementlike protein TEP1 function, and have other important roles in innate immune defence [40]. Information on all transcripts (TRINITY_DN0000_c00_g00_i00) are summarised in Additional file 2: Tables S2-S5.

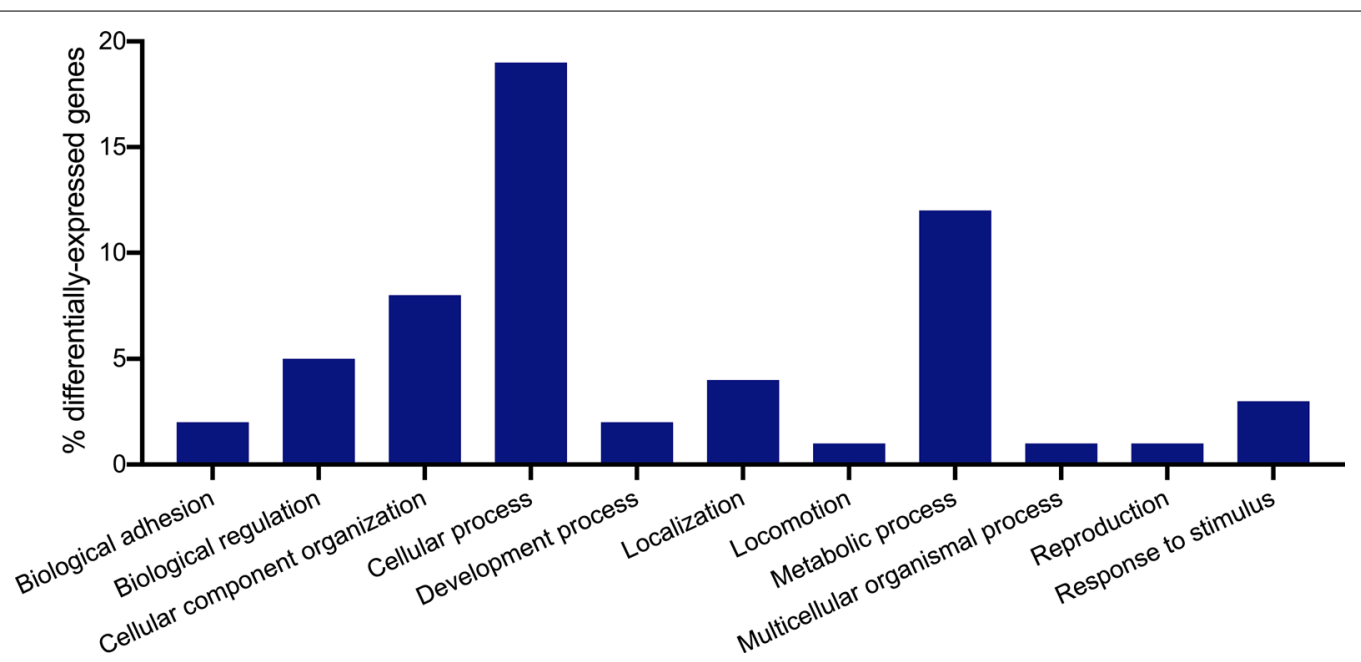

Fig. 4 Functional classification of Anopheles aquasalis midgut transcripts using gene ontology analysis. Summary of the general distribution of differentially expressed genes [\%] on PANTHER (http://www.pantherdb.org) 


\section{Detoxification}

Plasmodium vivax is probably able to modulate detoxification of free radicals while invading the midgut of An. aquasalis, as suggested by the increase of $\mathrm{H}_{2} \mathrm{O}_{2}$ following artificial reduction of catalase activity which leads to increased parasite infection in the mosquito midgut. As gene silencing also decreases the midgut microbiome, Bahia et al. [41] suggest that this manipulation occurs through the control of competitive bacteria which allows better parasite development. Transcript coding for CYP315A1 (TRINITY_DN6118_ c3_g2_i14) was upregulated in the $P v \times B l$ group, as was its orthologue in deltamethrin-resistant $A n$. gambiae mosquitoes when compared with a sensitive mosquito line from Kenya [42]. NAD+ kinase (TRINITY_DN5956_c5_g5_i5) and senecionine N-oxygenase (TRINITY_DN5975_c0_g1_i2) were also upregulated with infection, which suggests that the enzyme activity was needed for the antioxidant activities of other enzymes. Autophagic responses can be modulated by radical species and NAD+ homeostasis and the midgut metabolism can be an important player in autophagy regulation [43].

\section{Cytoskeleton remodelling}

Anopheles gambiae midgut response to P. berghei ookinete invasion is characterized by profound alterations in the transcription of genes that modulate the architecture of the cytoskeleton [38]. Plasmodium parasites need to modify the cytoskeleton of mosquito epithelial cells to successfully complete their life-cycle. We found several downregulated genes (TRINITY_DN4493_c0_g1, TRINITY_DN5277_c0_g1, TRINITY_DN5389_c0_g1, TRINITY_DN6055_c0_g1, TRINITY_DN6090_c5_g3, TRINITY_DN6296_c2_g1) that could be associated with cytoskeleton remodelling, which reinforces the prominent role of this cellular mechanism in response to Plasmodium and extends it to $P$. vivax ookinete invasion of the mosquito midgut.

\section{Autophagy}

The present transcriptional analysis suggested that differential expression of autophagic genes is involved in An. aquasalis females following a $P$. vivax infected blood meal. Forty-nine percent of differentially expressed genes during invasion $(60.0 \%$ of the upregulated and $44.1 \%$ of the downregulated genes) could be associated with autophagic processes (Table 2).

Table 2 Midgut differentially expressed genes associated with autophagy upon Plasmodium vivax invasion

\begin{tabular}{|c|c|c|}
\hline Transcript ID & Annotation & $\log F C$ \\
\hline TRINITY_DN1272_C0_g1_i2 & Protein spitz & 8.52 \\
\hline TRINITY_DN4493_C0_g1_i2 & T-complex protein 1 subunit gamma & -9.19 \\
\hline TRINITY_DN5389_c0_g1_i2 & gamma-tubulin complex component 5 & -8.22 \\
\hline TRINITY_DN5823_C0_g1_i1 & Facilitated trehalose transporter-Tret1 & -9.50 \\
\hline TRINITY_DN5851_C0_g1_i2 & GPCR family 2, methuselah receptor 6 & -3.20 \\
\hline TRINITY_DN5911_C0_g1_i4 & Beta-arrestin-1 & -9.97 \\
\hline TRINITY_DN5956_C5_g5_i5 & NAD kinase & 9.26 \\
\hline TRINITY_DN6021_C0_g3_i1 & OTU domain-containing protein 5 & -9.38 \\
\hline TRINITY_DN6039_c0_g1_i17 & Ubiquitin-like-conjugating enzyme ATG3 & -9.27 \\
\hline TRINITY_DN6055_c0_g1_i13 & Formin-like protein CG32138 & -11.08 \\
\hline TRINITY_DN6077_C7_g2_i1 & Guanylate cyclase $32 \mathrm{E}$ & -8.56 \\
\hline TRINITY_DN6090_c5_g3_i2 & Probable actin-related prot. 2/3 complex subunit2 & -8.39 \\
\hline TRINITY_DN6177_c2_g2_i3 & Trehalose-6-phosphate synthase/phosphatase & 8.01 \\
\hline TRINITY_DN6296_c2_g1_i5 & Plastin-3 & -9.74 \\
\hline TRINITY_DN6321_CO_g2_i2 & Endothelin-converting enzyme 1 & 8.43 \\
\hline TRINITY_DN6330_C2_g6_i2 & Fat storage-inducing transmembrane protein & -8.49 \\
\hline TRINITY_DN6333_c5_g2_i4 & Integrin beta-PS & 8.32 \\
\hline TRINITY_DN6454_c2_g2_i6 & Transcription factor kayak/AP-1 & 10.47 \\
\hline TRINITY_DN6473_c3_g4_i6 & Dynein light chain 1, cytoplasmic & 10.67 \\
\hline TRINITY_DN6489_c3_g1_i5 & Prominin & 3.40 \\
\hline TRINITY_DN6531_C1_g1_i4 & Sphingomyelin phosphodiesterase & 9.45 \\
\hline TRINITY_DN6536_c2_g8_i1 & Ubiquitin carboxyl-terminal hydrolase 10-A & -8.93 \\
\hline TRINITY_DN6571_c0_g10_i7 & Forkhead box protein $\mathrm{K} 2$ & -10.44 \\
\hline TRINITY_DN6646_c8_g1_i10 & Vesicle-trafficking protein SEC22b-B & -6.81 \\
\hline
\end{tabular}


Transcript coding for the GPCR Methuselah receptor 6 (TRINITY_DN5851_c0_g1_i2) was downregulated, as was $\beta$-arrestin (TRINITY_DN5911_c0_g1), which uncouples GPCRs from their G-proteins, and suggests that regulation of free radical production might occur through this molecule. In Drosophila, Methuselah receptors have been associated with lifespan and resistance to starvation and free radicals [44]. Wang et al. [45], using a specific agonist and antagonist, demonstrated that the TOR pathway is one of the major effectors underlying Methuselah. Blocking Methuselah reduced dTOR activity and promoted autophagy.

Trehalose is a natural sugar found in prokaryotes, yeast, fungi, plants and invertebrates, and serves not only as a reserve of carbohydrate, but can also protect organisms and cells against adverse environmental conditions. Some controversy exists on the real effect of trehalose on autophagy. In murine models, trehalose seems to induce autophagy, while in cultured cells it could inhibit fusions of autophagosomes and lysosomes, thus blocking the final stage of autophagy [46]. Our data suggest that the $A n$. aquasalis midgut increases intracellular trehalose by upregulating trehalose 6-phosphate synthase/phosphatase (TRINITY_DN6177_c2_g2) and downregulating the TreT1-facilitated trehalose transporter (TRINITY DN5823_c0_g1), suggesting autophagy induction in the An. aquasalis midgut during $P$. vivax infection. Anopheles gambiae TreT1 RNA silencing reduces the number of P. falciparum oocysts in the mosquito midgut [47], suggesting it might exert parasite protection during midgut invasion.

Microtubules (MT) are important to autophagosome formation and motility. Dynein light chain 1 (TRINITY DN6473_c3_g4), a motor protein, was upregulated upon infection in our study. In vertebrates, Beclin-1 is sequestered in MT in complexes containing dynein light chain 1. When autophagy is stimulated, Beclin-1 is released from this complex. In parallel, c-Jun N-terminal kinase-1 (JNK1) is activated which allows phosphorylation of Bcl-2 and Bim, which, in turn, releases Beclin 1 and contributes to autophagosome formation [48]. Regarding the gamma-tubulin complex component 5 (TRINITY_DN5389_c0_g1), involved in microtubule assembly [49], and T-complex protein 1 subunit gamma (TRINITY_DN4493_c0_g1), a chaperonin for tubulin and actin [50], we found that these were downregulated, possibly confirming that (as in other Anopheline species) microtubule dynamics are altered during $P$. vivax invasion of the midgut epithelium, and it is possible that this is associated to autophagy.

JNK signalling has been demonstrated to be involved in lifespan control and is required in differentiated cells of the intestinal epithelium in order to prevent excessive sensitivity of these cells to oxidative stress in Drosophila [51] and has been implicated in mosquito defense against malaria parasites, and altered expression patterns of autophagy biomarkers [22]. Garver et al. [52] showed that basal mRNA expression of the genes involved in JNK signalling were upregulated in the mosquito midgut and JNK silencing significantly increases the prevalence of infection. However, more recently, Souvannaseng et al. [53] demonstrated that moderate inhibition of JNK signaling in the An. stephensi midgut extended lifespan and enhanced resistance to P. falciparum. In the present study, we found that the AP-1 transcription factor (TRINITY_DN6454_c2_g2), a downstream product of this signalling pathway, was upregulated in mosquitoes fed on P. vivax blood.

The ubiquitin machinery regulates fundamental biological processes within eukaryotic cells. The enrichment of functional terms such as ubiquitin-dependent proteasome was also denoted for insects facing dehydration stress [54]. Nitric oxide synthase expression and nitric oxide increase in the midgut of An. aquasalis, An. stephensi and An. gambiae during Plasmodium parasite infection, which limits parasite development within the mosquito [55-57]. Ubiquitin carboxyl-terminal hydrolase (TRINITY_DN6536_c2_g8), ubiquitin-like-conjugating enzyme ATG3 (TRINITY_DN6039_c0_g1) and OTU domain-containing protein 5 (TRINITY_DN6021_c0_g3) were downregulated during midgut invasion by $P$. vivax. OTU domain-containing proteins are deubiquitinating enzymes and cleave distinct sets of ubiquitin chain types [58]. In vertebrates, OTU domain-containing protein 5 regulates interferon signalling [59]. USP10 regulates the deubiquitination of Beclin1 in Vps34 complexes, which in turn leads to a reduction in the levels of PtdIns3P and consequent inhibition of autophagy. Spautin-1, an inhibitor of USP10 and USP13, promotes the ubiquitination and degradation of Vps34 complexes, which in turn leads to a reduction in the levels of PtdIns3P and consequent inhibition of autophagy [60]. Since ubiquitination and deubiquitination are central to autophagy regulation, once more, our data indicated that $P$. vivax invasion of An. aquasalis midgut epithelium triggers an autophagic response. ATG3, among other enzymes, is involved in the maturation of the growing autophagosome, a process that occurs once autophagy is initiated. Recently, Frudd et al. [61] described a mechanism that associates reactive species to autophagy induction. The oxidation of Atg3 and Atg7 prevents phosphatidylethanolamine conjugation to LC3 (microtubule-associated protein 1A/1B-light chain 3 ), thus, associating reactive species to autophagy induction. The production of reactive oxygen/nitrogen species during ookinete invasion of An. aquasalis [41], together with the set of differentially displayed genes such as, is 
suggestive of an interplay between reactive species and autophagy during ookinete invasion of the midgut.

Overexpression of prominin 1 constitutively activates autophagy in the human retinal pigment epithelium via inhibition of mTORC1 and mTORC2, while it impairs autophagy via upregulation of mTORC1/2 activities. Prominin (TRINITY_DN6489_c3_g1) was upregulated during parasite invasion of the midgut epithelium, which suggests that autophagy might be activated during this stage of infection.

Lipid droplet (LD) homeostasis [62] also plays an important role in autophagy regulation. We observed that a fat storage-inducing transmembrane protein (TRINITY_DN6330_c2_g6), that was described to facilitate proper LD budding from the ER [63], was downregulated. Sphingolipids have also been associated with lipid droplet formation, and sphingomyelin phosphodiesterase 1 (TRINITY_DN6531_c1_g1) was upregulated. Although sphingomyelinases do not play a role in autophagy induction, the upregulation of sphingomyelin phosphodiesterase 1 (TRINITY_DN6531_c1_g1) can act in the autophago-lysosomal degradation [64], thus regulating the autophagosome formation. Vesicle transport protein SEC22 (TRINITY_DN6646_c8_g1), a protein implicated in autophagosome biogenesis [65], was downregulated, while endothelin-converting enzyme (TRINITY_DN6321_c0_g2), previously detected in autophagic vesicles [66], was upregulated. Regulation of these genes suggests that $P$. vivax invasion affects regulation of different stages of the autophagic process and includes autophagosome maturation and degradation.

Plasmodium invasion of the midgut epithelial cell leads to a number of molecular and morphological changes, including cell death. Vlachou et al. [67] proposed that the first invaded cells undergo apoptosis and are expelled to the lumen, while adjacent cells extend lamellipodia to maintain epithelium continuity. The ookinete reinvades several cells until it reaches the extracellular matrix in order to develop into oocysts, which implies a substantial dynamic rearrangement of the actin cytoskeleton. Division of regenerative cells within the midgut epithelium of an adult female An. stephensi in response to $P$. falciparum invasion [68] has been reported. Even so, the extent of apoptosis and the mechanisms by which the integrity of the midgut epithelium is maintained are not yet understood. The detachment-induced apoptosis (anoikis), driven by these morphological changes, can be deleterious to the mosquito and can be compensated by extracellular matrix (ECM) detachment, which induces autophagy [69] mediated by integrin [70]. Integrin are cell surface proteins that interact with the external cellular matrix (ECM), and signal through the cell membrane in both directions. Plasmodium vivax infection of the midgut positively regulated the expression of this gene (TRINITY_DN6333_c5_g2).

The Drosophila epidermal growth factor receptor (EGFR) pathway has been implicated in the control of delamination and anoikis of damaged enterocytes following oral bacterial infection [71] and Serratia marcescens infection of An. gambiae activates the EGFR pathway by modulating the outcome, possibly through synergistic functions in gut homeostasis [72]. Spitz (TRINITY_DN1272_c0_g1), the ligand of EGFR, was upregulated with infection and Forkhead box K2 (TRINITY_DN6571_c0_g10_i7) which can inhibit EGRF in 769-P cells [73], which suggests that the EGFR pathway is activated following $P$. vivax infection. This probably contributes to gut hemostasis through autophagy. Subcellular localization of the EGFR seems to be determinant on the effect on autophagy, being either an inhibitor or stimulant [74].

\section{Treatment of mosquitoes with autophagy inhibitor}

In order to evaluate the autophagy effect on the outcome of $P$. vivax infection in An. aquasalis, the mosquitoes were treated prior to infection with the autophagy inducer spermidine, or an autophagy inhibitor wortmannin.

When mosquitoes were treated with the autophagy inducer spermidine, the infection prevalence (IP) and infection intensity (II) were significantly lower: IP: Mann-Whitney U-test: $U=10196, P<0.0001$; II: Unpaired t-test: $\left.t_{(12)}=3.913, P=0.0021\right)$. A reduction of $44.9 \%$ (58.6 to $32.3 \%$ ) in IP and of $47 \%$ in II (25.7 to $13.6 \%)$ was observed. Wortmanin treatment resulted in a $54.3 \%$ reduction in IP and a $65 \%$ reduction in II when higher doses were used, while the $0.05 \mu \mathrm{M}$ doses resulted in a low reduction (7.9\%) of IP and a $5.9 \%$ increase in II (IP: Mann-Whitney U-test: W-5 $\mu \mathrm{M}^{*}$ control, $U=1351$, $P=0.0002$; W-0.05 $\mu \mathrm{M}^{*}$ control, $\left.U=7000, P=0.2357\right)$; II: t-test: W-5 $\mu \mathrm{M}^{*}$ control, $t_{(4)}=2,528, P=0.0648 ; \mathrm{W}-0.05$ $\mu \mathrm{M}^{*}$ control, $t_{(12)}=0.4003, P=0.6960$ ) (Fig. 5).

The differences between the two doses of wortmannin are probably a consequence of the drug mode of action. Wortmannin is a PI3-kinase inhibitor, therefore, as autophagosome formation requires class III PI3kinase activity, it is normally used to study the effect of autophagy inhibition. Nonetheless, wortmannin can also inhibit class I PI3-kinase activity (which inhibits autophagy) and can also inhibit mTOR (an autophagyinhibitory molecule) [75]. Furthermore, wortmannin can also act on the parasite and interfere with its development [76].

Spermidine is a polyamine that stimulates autophagy, both through mTOR-independent or dependent mechanisms [77] and its administration to mosquitoes 


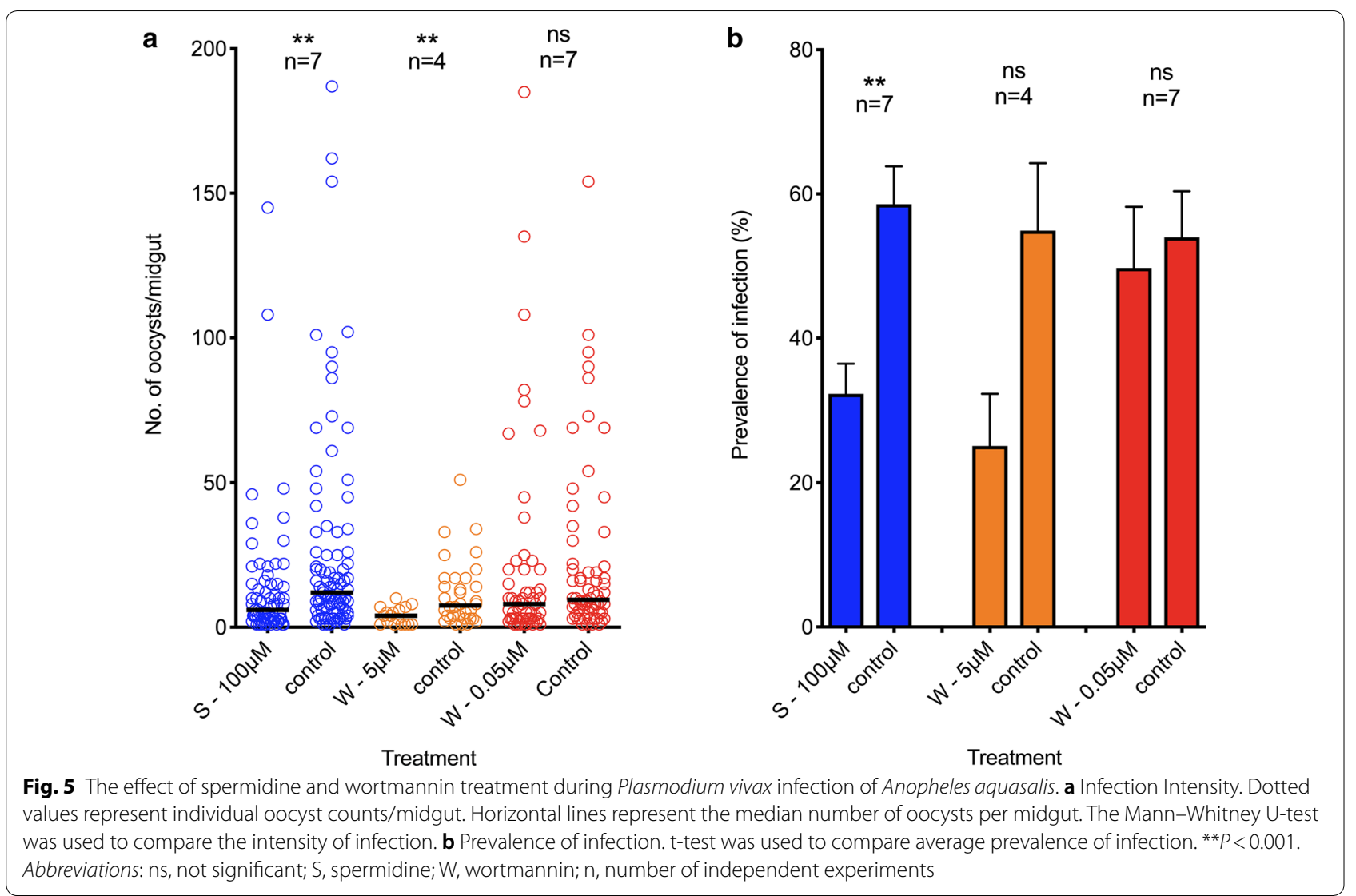

produces a significant reduction in $P$. vivax infection after treatment. Polyamine biosynthesis inhibitors cause growth arrest of $P$. falciparum blood stages in vitro but show no effect on survival of mice infected with $P$. berghei (reviewed in [78]). Despite these data, polyamide biosynthesis seems to be fundamental for sporogonic cycle completion. Targeted deletion of the enzyme AdoMetDC/ODC from Plasmodium yoelii blocks transmission to the mosquito An. stephensi, which could not be rescued by supplementation with spermidine [79]. This information reinforces that spermidine is acting on the mosquito rather than the parasite. Our data demonstrated a significant reduction in $P$. vivax infection after spermidine treatment in An. aquasalis, which, together with the data obtained using low wortmannin treatment, suggests that autophagy can control $P$. vivax infection in An. aquasalis.

\section{Expression of autophagy genes following Plasmodium vivax infection and treatment with autophagy suppressor and inhibitor}

To further characterize the role of autophagy in the mosquitoes treated with autophagy inhibitor in response to Plasmodium infection, qRT-PCR was used to quantify the changes in gene expression in response to a $P$. vivaxinfected blood meal. A differential expression analysis of several autophagy genes, including DRAM, Apg8 and Beclin, during inhibition and activation of autophagy, was performed. Atg8 protein, formerly known as Apg8/Aut7 is part of a group of proteins that control autophagy, many of which also participate in direct cytoplasmto-vacuole transport of proteins [80, 81]. Among the genes that promote autophagy is the damage-regulated autophagy modulator (DRAM-1), which belongs to an evolutionarily conserved family of proteins that encodes for a lysosomal protein that is required in order to induce autophagy [82, 83], and Beclin-1, which is part of a Class III phosphatidylinositol 3-kinase complex that is thought to be important in mediating localization of other Apg proteins to pre-autophagosomal structures [60].

No major differences were observed in expression of these genes after treatment with both drugs when compared with infection without treatment (Fig. 6). This is in-line with RNAseq data where transcription alterations of these genes were not detected. The major difference in expression was observed for beclin, which was downregulated after mosquitoes were treated with spermidine $(P=0.0635)$, suggesting that spermidine is 


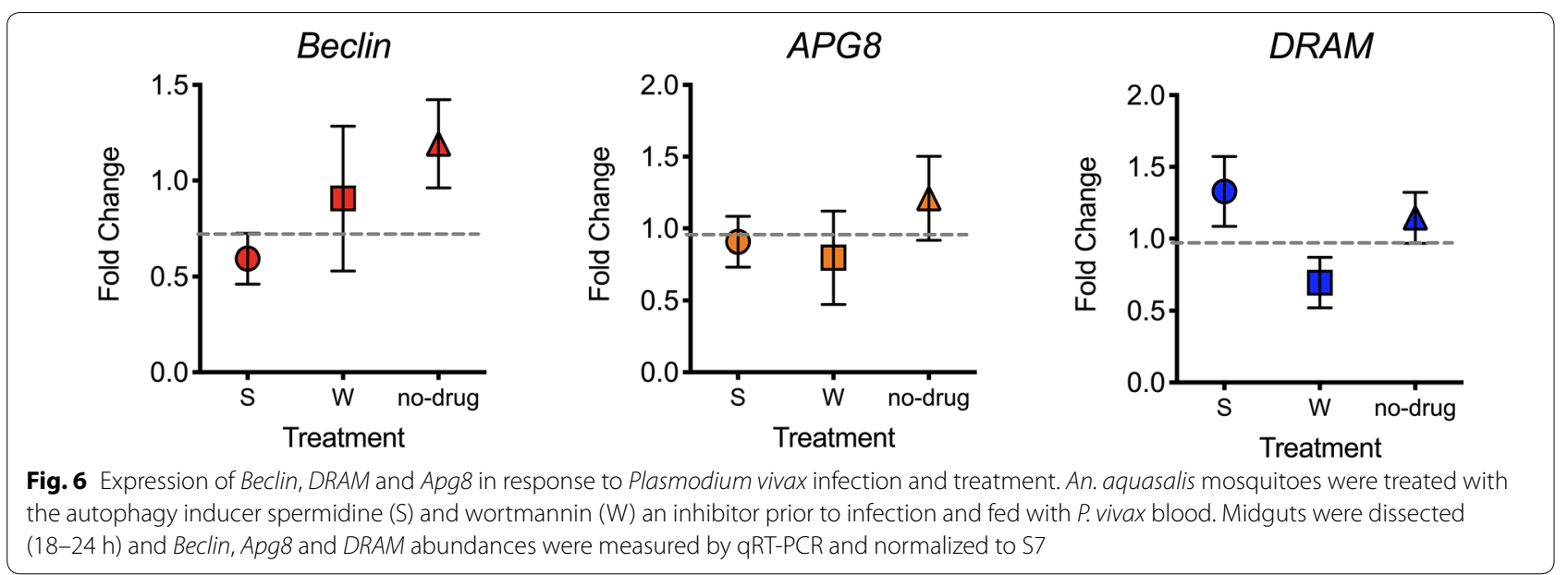

downregulating this gene while exerting a negative effect on $P$. vivax sporogonic development.

\section{Conclusions}

Our results clearly indicate that autophagy is regulated by P. vivax invasion of the mosquito midgut epithelium. A vast number of genes associated with autophagy were regulated by infection of which $60 \%$ were upregulated. Furthermore, when autophagy was inhibited by spermidine, we observed a significant reduction of the prevalence and intensity of infection. In view of our results, we propose that when ookinetes invade the midgut cells they trigger host cell morphological rearrangement, with actin and microtubule remodelling and production of nitrogen and oxygen radicals and possible cell death. To counterbalance invaded epithelial cell death/extrusion and other injuries parasites, could trigger an autophagic mechanism that would restrain parasite development, possibly through GPCR signalling Methuselah, the increase of intracellular trehalose, and detachment from the excellular matrix. This effect was apparent by the regulation of genes that could be assigned to the different stages of autophagy (initiation, nucleation, elongation/closure and maturation degradation) [84]. Autophagy triggered by Plasmodium invasion in epithelial midgut cells is a novel mechanism for mosquitos in order to fight Plasmodium infection.

\section{Additional files}

Additional file 1: Table S1. List of primers used in qRT-PCR analysis.

Additional file 2: Table S2. Lists of differentially transcribed genes $P \vee \times B I \_F D R<0.05$. Table S3. Lists of differentially transcribed genes $P \vee \times$ Unf_FDR $<0.05$. Table S4. Lists of differentially transcribed genes $\mathrm{P} \vee \times \mathrm{BI}$ _Total. Table S5. Lists of differentially transcribed genes P $\times \times$ Unf_Total.
Additional file 3: Figure S1. Validation of RNAseq analysis using qRT-PCR. Gene expression values for eight genes obtained by RNAseq were plotted against the corresponding averages of three qRT-PCR-derived gene expression values from biological replicates. The Pearson's correlation coefficient (0.874) and the best-fit linear-regression analysis $R^{2}=0.7663$ demonstrated a good degree of correlation between gene expression determined by each assay. Genes used for validation: TRINITY_DN4493_ C0_g1_i2, TRINITY_DN5277_c0_g1_i2, TRINITY_DN5911_CO_g1_i4, TRINITY_DN6055_C0_g1_i13, TRINITY_DN6039_C0_g1_i17, TRINITY_DN6296_ c2_g1_i5, TRINITY_DN6531_c1_g1_i4, TRINITY_DN6536_c2_g8_i1.

\section{Abbreviations}

RNAseq: RNA sequencing; cDNA: complementary DNA; WHO: World Health Organization; ATG: translation of autophagy; MFA: membrane feeding assay; DMSO: dimethyl sulfoxide; PBS: phosphate buffered saline; RT-qPCR: quantitative reverse transcription PCR.

\section{Acknowledgements}

We would like to thank all malaria vivax patients, healthy volunteers and the technical staff from the Malaria and Entomology Department of Fundação de Medicina Tropical Doutor Heitor Viera Dourado and the technical staff from Global Health and Tropical Medicine (GHMT), Universidade Nova de LisboaInstituto de Higiene e Medicina Tropical (IHMT-NOVA) Lisboa, Portugal. We acknowledge the financial support of Fundação para a Ciência e Tecnologia (GHTM-UID/Multi/04413/201) FAPEAM, Brazil, (Edital 030/2013-Universal Amazonas, 19716.UNI472.2459.20022014) and CAPES-Programa Ciência Sem Fonteiras (182/2012). To Universal-FAPEAM, CAPES-FAPEAM and CAPES-CSF for supporting publication fees.

\section{Authors' contributions}

RAGS contributed to the study design, performed experiments, analysed data and co-wrote the draft-manuscript. MCO, RCAS, DRTS and LF contributed to the experiments. MVGL and WMM contributed to data analysis, critical review of the manuscript and material resources. PA contributed to the bioinformatic analysis and critical review of manuscript. MGVBG contributed to the study design and data analysis, critical review of manuscript and financial resources. HS formulated the original hypothesis, drove the study design, contributed to data analyses, co-wrote the manuscript and financial resources. All authors read and approved the final manuscript.

\section{Funding}

This project was funded by the Amazonas State Research Foundation (Fundação de Amparo a Pesquisa do Estado do Amazonas, FAPEAM) via Edital 030/2013-Universal Amazonas, ref: 19716.UNI472.2459.20022014 and by Coordination for the Improvement of Higher Education Personnel (CAPES) through the Programme Science Without Borders. (182/2012). HS is a recipient of a Visiting Researcher Grant at the Fundação Medicina Tropical Heitor Vieira 
Dourado, Manaus, Brazil. HS acknowledges the financial support of Fundação para a Ciência e Tecnologia (GHTM-UID/Multi/04413/201).

\section{Availability of data and materials}

The datasets supporting the conclusions of this article are included within the article and its additional files.

\section{Ethics approval and consent to participate}

This study was approved by the Fundação de Medicina Tropical Dr. Heitor Vieira Dourado (FMT-HVD) Ethics Review Board (approval number: 422.289 CAAE: 20267013.8.0000.0005). All subjects provided signed written informed consent.

\section{Consent for publication}

Not applicable.

\section{Competing interests}

The authors declare that they have no competing interests.

\begin{abstract}
Author details
${ }^{1}$ Programa de Pós-Graduação em Medicina Tropical, Universidade do Estado do Amazonas/Fundação de Medicina Tropical Dr. Heitor Vieira Dourado, Manaus, Brazil. ${ }^{2}$ Instituto Leônidas \& Maria Deane, Fundação Oswaldo Cruz, Manaus, Brazil. ${ }^{3}$ Instituto de Higiene e Medicina Tropical, Global Health and Tropical Medicine, Universidade Nova de Lisboa, Lisboa, Portugal.
\end{abstract}

Received: 6 December 2018 Accepted: 14 May 2019

Published online: 24 May 2019

\section{References}

1. WHO. World malaria report 2017. Geneva: World Health Organization; 2017.

2. Ferreira MU, Castro MC. Challenges for malaria elimination in Brazil. Malar J. 2016;15:284.

3. Siqueira AM, Mesones-Lapouble O, Marchesini P, Sampaio VDS, Brasil P, Tauil PL, et al. Plasmodium vivax landscape in Brazil: scenario and challenges. Am J Trop Med Hyg. 2016;95:87-96.

4. Da Silva ANM, Santos CCB, Lacerda RN, Machado RLD, Póvoa MM. Susceptibility of Anopheles aquasalis and An. darlingi to Plasmodium vivax VK210 and VK247. Mem Inst Oswaldo Cruz. 2006;101:547-50.

5. Pimenta PFP, Orfano AS, Bahia AC, Duarte APM, Ríos-Velásquez CM, Melo $\mathrm{FF}$, et al. An overview of malaria transmission from the perspective of Amazon Anopheles vectors. Mem Inst Oswaldo Cruz. 2015;1 10:23-47.

6. Rios-Velásquez CM, Martins-Campos KM, Simões RC, Izzo T, Dos Santos EV, Pessoa FA, et al. Experimental Plasmodium vivax infection of key Anopheles species from the Brazilian Amazon. Malar J. 2013;12:460.

7. Sinden RE. Molecular interactions between Plasmodium and its insect vectors. Cell Microbiol. 2002;4:713-24.

8. Abraham EG, Jacobs-Lorena M. Mosquito midgut barriers to malaria parasite development. Insect Biochem Mol Biol. 2004;34:667-71.

9. Smith RC, Vega-Rodríguez J, Jacobs-Lorena M. The Plasmodium bottleneck: malaria parasite losses in the mosquito vector. Mem Inst Oswaldo Cruz. 2014;109:644-61.

10. Dennison NJ, BenMarzouk-Hidalgo OJ, Dimopoulos G. MicroRNA-regulation of Anopheles gambiae immunity to Plasmodium falciparum infection and midgut microbiota. Dev Comp Immunol. 2015:49:170-8.

11. Cirimotich CM, Dong Y, Garver LS, Sim S, Dimopoulos G. Mosquito immune defenses against Plasmodium infection. Dev Comp Immunol. 2010;34:387-95

12. Ramphul UN, Garver LS, Molina-Cruz A, Canepa GE, Barillas-Mury C. Plasmodium falciparum evades mosquito immunity by disrupting JNKmediated apoptosis of invaded midgut cells. Proc Natl Acad Sci USA. 2015;112:1273-80.

13. Parzych KR, Klionsky DJ. An overview of autophagy: morphology, mechanism, and regulation. Antioxid Redox Signal. 2014;20:460-73.

14. Ryoo HD, Baehrecke EH. Distinct death mechanisms in Drosophila development. Curr Opin Cell Biol. 2010;22:889-95.

15. Scott RC, Juhász G, Neufeld TP. Direct induction of autophagy by Atg1 inhibits cell growth and induces apoptotic cell death. Curr Biol. 2007;17:1-11.
16. Moy RH, Cherry S. Antimicrobial autophagy: a conserved innate immune response in Drosophila. J Innate Immun. 2013;5:444-55.

17. Nakagawa I, Amano A, Mizushima N, Yamamoto A, Yamaguchi H, Kamimoto T, et al. Autophagy defends cells against invading Group A streptococcus. Science. 2004;306:1037-40.

18. Orvedahl A, Macpherson S, Sumpter R Jr, Tallóczy Z, Zou Z, Levine B. Autophagy protects against Sindbis virus infection of the central nervous system. Cell Host Microbe. 2010;7:115-27.

19. Shelly S, Lukinova N, Bambina S, Berman A, Cherry S. Autophagy plays an essential anti-viral role in Drosophila against vesicular stomatitis virus. Immunity. 2009;30:588-98.

20. Yordy B, Iwasaki A. Cell type-dependent requirement of autophagy in HSV-1 antiviral defense. Autophagy. 2013;9:236-8.

21. Ling YM, Shaw MH, Ayala C, Coppens I, Taylor GA, Ferguson DJP, et al. Vacuolar and plasma membrane stripping and autophagic elimination of Toxoplasma gondii in primed effector macrophages. J Exp Med. 2006;203:2063-71.

22. Drexler AL, Pietri JE, Pakpour N, Hauck E, Wang B, Glennon EKK, et al. Human IGF1 regulates midgut oxidative stress and epithelial homeostasis to balance lifespan and Plasmodium falciparum resistance in Anopheles stephensi. PLoS Pathog. 2014;10:e1004231.

23. Eng MW, van Zuylen MN, Severson DW. Apoptosis-related genes control autophagy and influence DENV-2 infection in the mosquito vector, Aedes aegypti. Insect Biochem Mol Biol. 2016;76:70-83.

24. Akinosoglou KA, Bushell ESC, Ukegbu CV, Schlegelmilch T, Cho JS, Redmond S, et al. Characterization of Plasmodium developmental transcriptomes in Anopheles gambiae midgut reveals novel regulators of malaria transmission. Cell Microbiol. 2015;17:254-68.

25. Vontas J, David JP, Nikout D, Hemingway J, Christophides GK, Louis C, et al. Transcriptional analysis of insecticide resistance in Anopheles stephensi using cross-species microarray hybridization. Insect Mol Biol. 2007;16:315-24.

26. Costa-da-Silva AL, Marinotti O, Ribeiro JMC, Silva MCP, Lopes AR, Barros MS, et al. Transcriptome sequencing and developmental regulation of gene expression in Anopheles aquasalis. PLoS Negl Trop Dis. 2014;8:e3005.

27. Ministério de Saúde do Brasil, Secretaria de Vigilância em Saúde Departamento de Vigilância Epidemiologica. Malaria's treatment in Brazil practical guide. Brasília: Ministério da Saúde; 2010. p. 36

28. Mendes AM, Awono-Ambene PH, Nsango SE, Cohuet A, Fontenille D, Kafatos FC, et al. Infection intensity-dependent responses of Anopheles gambiae to the African malaria parasite Plasmodium falciparum. Infect Immun. 2011;79:4708-15.

29. Haas BJ, Papanicolaou A, Yassour M, Grabherr M, Philip D, Bowden J, et al. De novo transcript sequence reconstruction from RNA-Seq: reference generation and analysis with Trinity. Nat Protoc. 2013;8:1494-512.

30. Langmead B, Trapnell C, Pop M, Salzberg SL. Ultrafast and memoryefficient alignment of short DNA sequences to the human genome. Genome Biol. 2009;10:R25.

31. Li B, Dewey CN. RSEM : accurate transcript quantification from RNA-Seq data with or without a reference genome. BMC Bioinform. 2011;12:323.

32. Robinson MD, Mccarthy DJ, Smyth GK. edgeR: a bioconductor package for differential expression analysis of digital gene expression data. Bioinformatics. 2010;26:139-40.

33. Mi H, Huang X, Muruganujan A, Tang H, Mills C, Kang D, et al. PANTHER version 11: expanded annotation data from Gene Ontology and Reactome pathways, and data analysis tool enhancements. Nucleic Acids Res. 2017:45:D183-9.

34. Silveira H, Gabriel A, Ramos S, Palma J, Felix R, Custódio A, et al. CpG-containing oligodeoxynucleotides increases resistance of Anopheles mosquitoes to Plasmodium infection. Insect Biochem Mol Biol. 2012;42:758-65.

35. Félix RC, Silveira $H$. The interplay between tubulins and $P 450$ cytochromes during plasmodium berghei invasion of Anopheles gambiae midgut. PLOS ONE. 2011;6:e24181.

36. Pietrocola F, Lachkar S, Enot DP, Pedro JMB, Sica V, Izzo V, et al. Spermidine induces autophagy by inhibiting the acetyltransferase EP300. Cell Death Differ. 2015;22:509-16.

37. Minois N, Carmona-Gutierrez D, Bauer MA, Rockenfeller P, Eisenberg T, Brandhorst $\mathrm{S}$, et al. Spermidine promotes stress resistance in Drosophila melanogaster through autophagy-dependent and -independent pathways. Cell Death Dis. 2012;3:e401-8. 
38. Vlachou D, Schlegelmilch T, Christophides GK, Kafatos FC. Functional genomic analysis of midgut epithelial responses in Anopheles during Plasmodium invasion. Curr Biol. 2005;15:1185-95.

39. Pinto SB, Lombardo F, Koutsos AC, Waterhouse RM, Mckay K, An C, et al. Discovery of Plasmodium modulators by genome-wide analysis of circulating hemocytes in Anopheles gambiae. Proc Natl Acad Sci USA. 2009;106:21270-5.

40. Le BV, Williams M, Logarajah S, Baxter RHG. Molecular basis for genetic resistance of Anopheles gambiae to Plasmodium: structural analysis of TEP1 susceptible and resistant alleles. PLoS Pathog. 2012;8:e1002958.

41. Bahia AC, Oliveira JHM, Kubota MS, Araújo HRC, Lima JBP, Ríos-Velásquez $\mathrm{CM}$, et al. The role of reactive oxygen species in Anopheles aquasalis response to Plasmodium vivax infection. PLoS ONE. 2013;8:e57014.

42. Bonizzoni M, Ochomo E, Dunn WA, Britton M, Afrane Y, Zhou G, et al. RNAseq analyses of changes in the Anopheles gambiae transcriptome associated with resistance to pyrethroids in Kenya: identification of candidateresistance genes and candidate-resistance SNPs. Parasit Vectors. 2015;8:474.

43. Zhang D-X, Zhang J, Hu J, Huang Y. The potential regulatory roles of NAD+ and its metabolism in autophagy. Metabolism. 2016;65:454-62.

44. Lin I, Seroude L, Benzer S. Extended life-span and stress resistance in the Drosophila mutant methuselah. Science. 1998;282:943-6.

45. Wang J, Wang Z, Zhang Z, Hua Q, Wang M, Shi C, et al. Methuselah regulates longevity via dTOR: a pathway revealed by small-molecule ligands. J Mol Cell Biol. 2015;7:280-2.

46. Lee H, Yoon Y, Lee S. Mechanism of neuroprotection by trehalose: controversy surrounding autophagy induction. Cell Death Dis. 2018;9:712.

47. Liu K, Dong Y, Huang Y, Rasgon JL, Agre P. Impact of trehalose transporter knockdown on Anopheles gambiae stress adaptation and susceptibility to Plasmodium falciparum infection. Proc Natl Acad Sci USA. 2013;1 10:17504-9.

48. Mackeh R, Perdiz D, Lorin S, Codogno P, Pous C. Autophagy and microtubules_new story, old players. J Cell Sci. 2013;126:1071-80.

49. Vleugel M, Kok M, Dogterom M. Understanding force-generating microtubule systems through in vitro reconstitution. Cell Adhes Migr. 2016;10:475-94

50. Sternlicht H, Farr GW, Sternlicht ML, Driscoll JK, Willisont K, Yaffe MB. The $\mathrm{t}$-complex polypeptide 1 complex is and actin in vivo chaperonin for tubulin and actin in vivo. Proc Natl Acad Sci USA. 1993:90:9422-6.

51. Biteau B, Hochmuth CE, Jasper H. JNK activity in somatic stem cells causes loss of tissue homeostasis in the aging Drosophila gut. Cell Stem Cell. 2008;3:442-55.

52. Garver LS, Oliveira GDA, Barillas-Mury C. The JNK pathway is a key mediator of Anopheles gambiae antiplasmodial immunity. PLoS Pathog. 2013;9:e1003622.

53. Souvannaseng L, Hun LV, Baker H, Klyver JM, Wang B, Pakpour N, et al. Inhibition of JNK signaling in the Asian malaria vector Anopheles stephensi extends mosquito longevity and improves resistance to Plasmodium falciparum infection. PLoS Pathog. 2018;14:e1007418.

54. Choy A, Severo MS, Sun R, Girke T, Gillespie JJ, Pedra JHF. Decoding the ubiquitin-mediated pathway of arthropod disease vectors. PLoS ONE. 2013:8:e78077.

55. Bahia AC, Kubota MS, Tempone AJ, Araujo HRC, Guedes BAM, Orfano AS, et al. The JAK-STAT pathway controls Plasmodium vivax load in early stages of Anopheles aquasalis infection. PLoS NegI Trop Dis. 2011;5:e1317.

56. Luckhart S, Odovotz Y, Cui L, Rosenberg R. The mosquito Anopheles stephensi limits malaria parasite development with inducible synthesis of nitric oxide. Proc Natl Acad Sci USA. 1998;95:5700-5.

57. Tahar R, Boudin C, Thiery I, Bourgouin C. Immune response of Anopheles gambiae to the early sporogonic stages of the human malaria parasite Plasmodium falciparum. EMBO J. 2002;21:6673-80.

58. Mevissen TET, Hospenthal MK, Geurink PP, Elliott PR, Akutsu M, Arnaudo $\mathrm{N}$, et al. OTU deubiquitinases reveal mechanisms of linkage specificity and enable ubiquitin chain restriction analysis. Cell. 2013;154:169-84.

59. Kayagaki N, Phung Q, Chan S, Chaudhari R, Quan C, Zhang Z, et al. DUBA: a deubiquitinase that regulates type I interferon production. Science. 2007:318:1628-32.

60. Liu J, Xia H, Kim M, Xu L, Li Y, Zhang L, et al. Beclin1 controls the levels of p53 by regulating the deubiquitination activity of USP10 and USP13. Cell. 2011;147:223-34.
61. Frudd K, Burgoyne T, Burgoyne JR. Oxidation of Atg3 and Atg7 mediates inhibition of autophagy. Nat Commun. 2018;9:95.

62. Dong H, Czaja MJ. Regulation of lipid droplets by autophagy. Trends Endocrinol Metab. 2011;22:234-40.

63. Choudhary V, Ojha N, Golden A, Prinz WA. A conserved family of proteins facilitates nascent lipid droplet budding from the ER. J Cell Biol. 2015;211:261-71.

64. Perrotta C, Cervia D, De Palma C, Assi E, Pellegrino P, Teresa M, et al. The emerging role of acid sphingomyelinase in autophagy. Apoptosis. 2015;20:635-44.

65. Amaya C, Fader CM, Colombo MI. Autophagy and proteins involved in vesicular trafficking. FEBS Lett. 2015;589:3343-53.

66. Pacheco-Quinto J, Eckman EA. Endothelin-converting enzymes degrade intracellular $\beta$-amyloid produced within the endosomal/lysosomal pathway and autophagosomes. J Biol Chem. 2013;288:5606-15.

67. Vlachou D, Zimmermann T, Cantera R, Janse CJ, Waters AP, Kafatos FC. Real-time, in vivo analysis of malaria ookinete locomotion and mosquito midgut invasion. Cell Microbiol. 2004;6:671-85.

68. Baton LA, Ranford-Cartwright LC. Morphological evidence for proliferative regeneration of the Anopheles stephensi midgut epithelium following Plasmodium falciparum ookinete invasion. J Invertebr Pathol. 2007;96:244-54.

69. Fung C, Lock R, Gao S, Salas E, Debnath J. Induction of autophagy during extracellular matrix detachment promotes cell survival. Mol Biol Cell. 2008;19:308-17.

70. Vlahakis A, Debnath J. The interconnections between autophagy and integrin-mediated cell adhesion. J Mol Biol. 2017:429:515-30.

71. Buchon N, Broderick NA, Kuraishi T, Lemaitre B. Drosophila EGFR pathway coordinates stem cell proliferation and gut remodeling following infection. BMC Biol. 2010;8:152

72. Stathopoulos S, Neafsey DE, Lawniczak MKN, Muskavitch MAT, Christophides GK. Genetic dissection of Anopheles gambiae gut epithelial responses to Serratia marcescens. PLoS Pathog. 2014;10:e1003897.

73. Zhang F, Ma X, Li H, Zhang Y, Li X, Chen L, et al. FOXK2 suppresses the malignant phenotype and induces apoptosis through inhibition of EGFR in clear-cell renal cell carcinoma. Int J Cancer. 2018;142:2543-57.

74. Li H, You L, Xie J, Pan H, Han W. The roles of subcellularly located EGFR in autophagy. Cell Signal. 2017;35:223-30.

75. Mizushima N, Yoshimorim T, Levine B. Methods in mammalian autophagy research. Cell. 2010;140:313-26.

76. Vaid A, Ranjan R, Smythe WA, Hoppe HC, Sharma P. PfPI3K, a phosphatidylinositol-3 kinase from Plasmodium falciparum, is exported to the host erythrocyte and is involved in hemoglobin trafficking. Blood. 2010;115:2500-7.

77. Moulis M, Vindis C. Methods for measuring autophagy in mice. Cells. 2017:6:14.

78. Phillips MA. Polyamines in protozoan pathogens. J Biol Chem. 2018;293:18746-56.

79. Hart RJ, Ghaffar A, Abdalal S, Perrin B, Aly AS. Plasmodium AdoMetDC/ ODC bifunctional enzyme is essential for male sexual stage development and mosquito transmission. Biol Open. 2016:5:1022-9.

80. Harding TM, Hefner-Gravink A, Thumm M, Klionsky DJ. Genetic and phenotypic overlap between autophagy and the cytoplasm to vacuole protein targeting pathway. J Biol Chem. 1996;271:17621-4.

81. Klionsky DJ, Cregg JM, Dunn WA Jr, Emr SD, Sakai Y, Sandoval IV, et al. A unified nomenclature for yeast autophagy-related genes. Dev Cell. 2003;5:539-45

82. Crighton D, Wilkinson S, O'Prey J, Syed N, Smith P, Harrison PR, et al. DRAM, a p53-induced modulator of autophagy, is critical for apoptosis. Cell. 2006:126:121-34.

83. Mah LY, O'Prey J, Baudot AD, Hoekstra A, Ryan KM. DRAM-1 encodes multiple isoforms that regulate autophagy. Autophagy. 2012;8:18-28.

84. Wong ASL, Cheung ZH, Ip NY. Molecular machinery of macroautophagy and its deregulation in diseases. Biochim Biophys Acta. 2011:1812:1490-7.

\section{Publisher's Note}

Springer Nature remains neutral with regard to jurisdictional claims in published maps and institutional affiliations. 